\title{
A Class of Fourth Order Approximate Solutions of Stationary Exterior Fields of Einstein-Maxwell Equations
}

\author{
${ }^{1} \mathrm{Md}$. Abdus Salam \\ ${ }^{1}$ (Institute of Education, Research \& Training (IERT), University of Chittagong, Chittagong-4331, Bangladesh)
}

\begin{abstract}
A class of approximate solutions of stationary exterior fields of Einstein-Maxwell (EM) equations are obtained by expanding the metric in powers of a certain parameter and solving explicitly the first few orders in terms of four harmonic functions. Previously these approximate solutions in closed form were found upto third order. In the present paper we obtain new fourth order equations and find their approximate solutions for the particular choice of the harmonic functions. The harmonic functions are so chosen that all the approximate solutions obtained are asymptotically flat. Here some relations obtained are claimed to be a 'laboratory' with which future attempts at exact solutions in terms of harmonic functions may be tested.
\end{abstract}

Keywords: Einstein-Maxwell equations, Physical Interpretation, Approximate Solutions.

\section{Introduction}

Earlier, we obtained the third order approximate solutions of stationary (rotating) exterior (vacuum) fields of Einstein-Maxwell (EM) equations in terms of four harmonic functions with brief discussions about asymptotic properties [1 -6]. Following similar procedures in this paper (hereinafter referred to as "Paper V"), we shall find a class of fourth order approximate solutions of stationary exterior EM equations in terms of explicit forms of four harmonic functions. Here we also briefly discuss some asymptotic properties [7,8].

Papapetrou [9] and Majumdar [10] independently in 1947, discovered electrostatic (non-rotating) solutions of the EM equations which are given in terms of a single harmonic function, have no spatial symmetry (i.e., they are non- axisymmetric), and are produced by sources with $m=|\mathrm{e}|, m$ and e being the mass and charge respectively in suitable units. We call these the PM solutions which are different from the Papapetrou [9] rotating solutions of the exterior field of Einstein's equations considered earlier. Weyl's [11] electrostatic (nonrotating) solutions of the EM equations have axial symmetry and the sources satisfy $m=\beta e$, where $\beta$ is a constant and is the same for all masses. These Weyl solutions are given in terms of a single axisymmetric harmonic function. Perjes [12] and independently Israel and Wilson [13] generalized the PM solutions to a class of stationary (rotating) solutions of the EM equations with no spatial symmetry which arise from sources satisfying

$$
m=|\mathrm{e}|, \mathbf{h}= \pm \boldsymbol{\mu},
$$

where $\mathbf{h}$ and $\boldsymbol{\mu}$ are respectively the angular momentum and magnetic moment vectors of the source. These are called PIW solutions and are expressed in terms of two harmonic functions. Bonnor [14] attempted to find a class of axially symmetric stationary solutions depending on two harmonic functions that is related to PIW solutions in the same way as the Weyl class is related to the PM solutions. This new class would be associated with source satisfying the relations :

$$
\mathrm{m}=\beta e, \mathbf{h}=\beta^{\prime} \boldsymbol{\mu},
$$

where $\beta^{\prime}$ is another constant. It was an unsuccessful attempt but Bonnor found a class of axisymmetric rotating solutions of the EM equations that is expressible in terms of a single harmonic function. This class is unphysical in a similar sense to that in which the Papapetrou rotating solutions of the exterior Einstein equations are unphysical [6,9], namely asymptotically flat solution can be got only by making the mass of the source zero.

Considering a power series expansion of the metric in terms of a certain parameter, we can construct a class of axisymmetric stationary solutions explicitly to the first few orders in terms of four harmonic functions. With suitable choice of the harmonic functions this class yields to the order considered, the Weyl solutions, the Bonnor solutions and the axisymmetric form of the PIW solutions respectively [3,5,6]. A class of approximate solutions of stationary exterior EM equations in terms of four harmonic functions has been studied by Islam [5,6], Salam [2,3], upto the second order. Explicit solutions in closed form in terms of particular choice of harmonic functions upto the third order has been studied by Salam [1,3]. Following the procedure of Salam [1] in this paper we solve the one more higher order, i.e., fourth order EM equations explicitly for particular choice of the harmonic functions [7].

In section II, we write down the field equations with some known solutions. In section III, we derive the fourth order EM equations and some approximate solutions. The physical interpretation is considered in section IV, and higher order EM equations and their approximate solutions obtained in section V. 


\section{The Field Equations and Some Known Solutions}

The Einstein- Maxwell (EM) exterior equations, in suitable units are given by

$$
\begin{aligned}
& R_{\mu v}=8 \pi E_{\mu \nu}=-2 F_{\mu}{ }^{\alpha} F_{v \alpha}+\frac{1}{2} g_{\mu \nu} F_{\alpha \beta} F^{\alpha \beta}, \\
& F_{\mu v ; \sigma}+F_{v \sigma ; \mu}+F_{\sigma u ; v}=0, \\
& F_{; v}^{\mu \nu}=-4 \pi J^{\mu}=0, F_{\mu v}=A_{\mu, v}-A_{v, \mu},
\end{aligned}
$$

where $E_{\mu \nu}$ is the electromagnetic energy-momentum tensor, and $J^{\mu}$ the four- current which we put equal to zero, since we consider the exterior field only; $F_{\mu \nu}$ is the electromagnetic field tensor, defined in terms of the four-vector potential $A_{\mu}$ by (3c). A semicolon denotes covariant differentiation and a comma partial differentiation. Greek indices take the values $0,1,2,3$ and the repeated Greek indices are to be summed over these values. The tensor $F_{\mu \nu}$ satisfies (3b) identically with its definition in terms of $A_{\mu}$. Equation (3a) follows from the Einstein's equations

$$
R_{\mu \nu}-\frac{1}{2} g_{\mu \nu} R=8 \pi T_{\mu \nu},
$$

where $R_{\mu \nu}$ is the Ricci tensor, $g_{\mu \nu}$ is the metric tensor. If we interpret $T_{\mu \nu}$ as $E_{\mu \nu}$, then the Ricci scalar $R$ vanishes identically since $E_{\mu}{ }^{\mu}=0$. By using standard procedure of Maxwell theory, it can also be shown that $E_{\mu \nu}$ has zero divergence:

$$
E_{; v}^{\mu v}=0,
$$

which represents the conservation of energy and momentum of the electromagnetic field [3,5,6,7]. The foregoing equations have been written down explicitly by Bonnor [14] for the standard form of a stationary axisymmetric metric, namely,

$$
d s^{2}=f(d t-w d \phi)^{2}-\rho^{2} f^{-1} d \phi^{2}-e^{\mu}\left(d \rho^{2}+d z^{2}\right),
$$

where $f, w$ and $\mu$ are all functions of $\rho$ and $z$ [3,6]. Writing

$$
\left(x^{0}, x^{1}, x^{2}, x^{3}\right)=(t, \rho, z, \phi), c=1,
$$

the vector potential $\left(A_{0}, A_{1}, A_{2}, A_{3}\right)$ can be written in terms of two scalar fields $\Phi$ and $\Phi^{\prime}$ and the metric functions $f$ and $w$ as follows [15]:

$$
\begin{aligned}
& A_{0}=\Phi, A_{1}=A_{2}=0, \frac{\partial A_{3}}{\partial \rho}=w \Phi_{\rho}+\rho f^{-1} \Phi_{z}^{\prime}, \\
& \frac{\partial A_{3}}{\partial z}=w \Phi_{z}-\rho f^{-1} \Phi_{\rho}^{\prime},
\end{aligned}
$$

where $\Phi_{\rho} \equiv \frac{\partial \Phi}{\partial \rho}$, etc. The consistency of the two relations in (7) is guaranteed by the following equation (8d).

The field equations (3a) and (3c) in the metric (6) and for $A_{\mu}$ given by (7) yield the following four equations $[1,3,5,6]$ :

$$
\begin{aligned}
& f \nabla^{2} f-f_{\rho}^{2}-f_{z}^{2}+\rho^{-2} f^{4}\left(w_{\rho}^{2}+w_{z}^{2}\right)=2 f\left(\Phi_{\rho}^{2}+\Phi_{z}^{2}+\Phi_{\rho}^{\prime 2}+\Phi_{z}^{\prime 2}\right), \\
& f \Delta w+2 f_{\rho} w_{\rho}+2 f_{z} w_{z}=4 \rho f^{-1}\left(\Phi_{z}^{\prime} \Phi_{\rho}-\Phi_{\rho}^{\prime} \Phi_{z}\right), \\
& f \nabla^{2} \Phi=f_{\rho} \Phi_{\rho}+f_{z} \Phi_{z}+\rho^{-1} f^{2}\left(w_{z} \Phi_{\rho}^{\prime}-w_{\rho} \Phi_{z}^{\prime}\right), \\
& f \nabla^{2} \Phi^{\prime}=f_{\rho} \Phi_{\rho}^{\prime}+f_{z} \Phi_{z}^{\prime}+\rho^{-1} f^{2}\left(w_{\rho} \Phi_{z}-w_{z} \Phi_{\rho}\right),
\end{aligned}
$$

where the operators $\nabla^{2}, \Delta$ are respectively defined by 


$$
\nabla^{2} \equiv\left(\frac{\partial^{2}}{\partial \rho^{2}}+\frac{\partial^{2}}{\partial z^{2}}+\rho^{-1} \frac{\partial}{\partial \rho}\right) \text { and } \Delta \equiv\left(\frac{\partial^{2}}{\partial \rho^{2}}+\frac{\partial^{2}}{\partial z^{2}}-\rho^{-1} \frac{\partial}{\partial \rho}\right)
$$

(Salam, 2010, Eqs (1.21a), 1.26a)respectively) [7].

When $\Phi=\Phi^{\prime}=0$, equations (8a,b) reduce to

$f \nabla^{2} f-f_{\rho}^{2}-f_{z}^{2}+\rho^{-2} f^{4}\left(w_{\rho}^{2}+w_{z}^{2}\right)=0$

and

$f \Delta w+2 f_{\rho} w_{\rho}+2 f_{z} w_{z}=0$ respectively [2,3].

The field equations yield the following two more equations for $\mu$ :

$$
\begin{aligned}
& \mu_{\rho}=-f^{-1} f_{\rho}+\frac{1}{2} \rho f^{-2}\left(f_{\rho}^{2}-f_{z}^{2}\right)+2 \rho f^{-1}\left(\Phi_{z}^{2}-\Phi_{\rho}^{2}+\Phi_{z}^{\prime 2}-\Phi_{\rho}^{\prime 2}\right)+\frac{1}{2} \rho^{-1} f^{2}\left(w_{z}^{2}-w_{\rho}^{2}\right), \\
& \mu_{z}=-f^{-1} f_{z}+\rho f^{-2} f_{\rho} f_{z}-4 \rho f^{-1}\left(\Phi_{\rho} \Phi_{z}+\Phi_{\rho}^{\prime} \Phi_{z}^{\prime}\right)-\rho^{-1} f^{2} w_{\rho} w_{z} .
\end{aligned}
$$

The consistency of $(9 \mathrm{a}, \mathrm{b})$ is guaranteed by $(8 \mathrm{a}-\mathrm{d})$. When $\Phi=\Phi^{\prime}=0$, (9a), (9b) reduce to

$$
\mu_{\rho}=-f^{-1} f_{\rho}+\frac{1}{2} \rho f^{-2}\left(f_{\rho}^{2}-f_{z}^{2}\right)+\frac{1}{2} \rho^{-1} f^{2}\left(w_{z}^{2}-w_{\rho}^{2}\right) \text {, }
$$

and

$$
\mu_{z}=-f^{-1} f_{z}+\rho f^{-2} f_{\rho} f_{z}-\rho^{-1} f^{2} w_{\rho} w_{z}
$$

respectively. The equations $(8 \mathrm{a}-\mathrm{d})$ are therefore the basic equations, since $\mu$ can be obtained by a trivial integration once $f, w, \Phi$ and $\Phi^{\prime}$ have been solved from ( $\left.8 \mathrm{a}-\mathrm{d}\right)$.

The Weyl solutions are given by

$$
f=h^{-1}=(\Phi+a)^{2}+b^{2}, \Phi=-a+b \tan \lambda \tau, \quad \Phi^{\prime}=0, w=0,
$$

where $a, b$ are arbitrary constants and $\tau$ is a harmonic function. For convenience the constants are written in such a way that $f$ is a positive definite quadratic in $\Phi$. For this case there is no essential loss of generality. Here a constant parameter $\lambda$ is introduced and in the other solutions for reasons that will emerge later.

The Bonnor solutions are given by

$$
\begin{aligned}
& f=h^{-1}=\alpha\left(1+u^{2}\right)^{-1}\left[(u-B)^{2}+A^{2}\right], \\
& \Phi=\beta\left(1+u^{2}\right)^{-1} u+\Phi_{0}, \Phi^{\prime}=-\frac{1}{2} \beta\left(1+u^{2}\right)^{-1}\left(1-u^{2}\right)+\Phi_{0}^{\prime}, \\
& w_{\rho}=\left(\frac{2 \lambda}{\alpha A}\right) \rho \sigma_{z}^{\prime}, \quad w_{z}=-\left(\frac{2 \lambda}{\alpha A}\right) \rho \sigma_{\rho}^{\prime},
\end{aligned}
$$

where $\alpha, \beta, A, B, \Phi_{0}, \Phi_{0}^{\prime}$ are arbitrary constants and $\beta^{2}=\alpha\left(1+A^{2}+B^{2}\right)$. The function $u$ is given in terms of the harmonic function $\sigma^{\prime}$ by

$u=A \tan \lambda \sigma^{\prime}+B[1,3]$.

The foregoing Bonnor's solution is slightly different from that given in Bonnor (1973) [14]. We have considered the case where his constants $a$ and $b$ satisfy $a^{2}+b^{2}<4$, the opposite case being essentially the same. When $a^{2}+b^{2}=4$, Bonnor's solution becomes a special case of the PIW solutions ; this fact is not apparent from $(11 \mathrm{a}-\mathrm{d})$ but is not important for the present. To facilitate comparison with the approximate solution, we have written $\lambda \sigma^{\prime}$ for $\sigma^{\prime}$, where $\lambda$ is a constant parameter.

The non-axisymmetric PIW solutions are given by

$$
d s^{2}=|U|^{-2}\left(d t+\Omega^{(1)} d x+\Omega^{(2)} d y+\Omega^{(3)} d z\right)^{2}-|U|^{2}\left(d x^{2}+d y^{2}+d z^{2}\right),
$$

where $U$ is any complex solution of Laplace's equation in the cartesian coordinates $x, y, z$ (i.e., $U$ is the form $U_{1}+i U_{2}$ where $U_{1}$ and $U_{2}$ are real non-axisymmetric harmonic functions), and the vector $\boldsymbol{\Omega}=\left(\Omega^{(1)}, \Omega^{(2)}, \Omega^{(3)}\right)$ is found by solving the equation 
$\nabla \wedge \Omega=i\left(U \nabla U^{*}-U^{*} \nabla U\right)$,

where $U^{*}$ is the complex conjugate of $U$. The electromagnetic field can be described is terms of nonaxisymmetric potentials $\Phi, \Phi^{\prime}$ by

$\Phi+i \Phi^{\prime}=1 / U=U^{*} /||^{2}$.

To obtain the axisymmetric form of the solution, let

$\Omega^{(3)}=0, \rho^{2} \Omega^{(1)}=y w, \rho^{2} \Omega^{(2)}=-x w, x=\rho \cos \phi, y=\rho \sin \phi$,

and taking $U=\eta+i \xi$, where $\eta$ and $\xi$ are axisymmetric harmonic functions. With the foregoing condition (13), the metric (12) reduces to (6) and we get the axisymmetric form of the PIW solutions as follows :

$f=\Phi^{2}+\Phi^{\prime 2}=\left(\eta^{2}+\xi^{2}\right)^{-1}$

$\Phi=\left(\eta^{2}+\xi^{2}\right)^{-1} \eta, \Phi^{\prime}=-\left(\eta^{2}+\xi^{2}\right)^{-1} \xi$,

$w_{\rho}=2 \rho\left(\xi \eta_{z}-\eta \xi_{z}\right), w_{z}=-2 \rho\left(\xi \eta_{\rho}-\eta \xi_{\rho}\right)$,

where in (16c), we have used $\frac{\partial \eta}{\partial x}=\rho^{-1} x \eta_{\rho}$, etc. The desired form of the PIW solutions are respectively obtained from $(16 \mathrm{a}-\mathrm{c})$ by setting $\eta=\lambda \xi^{\prime}+1$ and $\xi=\lambda \eta^{\prime}$, which is permissible since $\lambda \xi^{\prime}+1$ and $\lambda \eta^{\prime}$ are harmonic, assuming that $\xi^{\prime}, \eta^{\prime}$ are harmonic functions.

$f=h^{-1}=\left(\eta^{2}+\xi^{2}\right)^{-1}$

$\therefore f^{-1}=h=\left(\lambda \xi^{\prime}+1\right)^{2}+\lambda^{2} \eta^{\prime 2}$,

$\left.\begin{array}{l}\Phi=\left(\eta^{2}+\xi^{2}\right)^{-1} \eta=\frac{\lambda \xi^{\prime}+1}{\left(\lambda \xi^{\prime}+1\right)^{2}+\lambda^{2} \eta^{\prime 2}} \\ \Phi^{\prime}=-\frac{\xi}{\left(\eta^{2}+\xi^{2}\right)}=-\frac{\lambda \eta^{\prime}}{\left(\lambda \xi^{\prime}+1\right)^{2}+\lambda^{2} \eta^{\prime 2}}\end{array}\right\}$,

$w_{\rho}=2 \rho\left(\xi \eta_{z}-\eta \xi_{z}\right)$

$=2 \rho\left[\lambda \eta^{\prime} \lambda \xi_{z}^{\prime}-\left(\lambda \xi^{\prime}+1\right) \lambda \eta_{z}^{\prime}\right]=-2 \rho\left[\lambda \eta_{z}^{\prime}\left(\lambda \xi^{\prime}+1\right)-\lambda^{2} \eta^{\prime} \xi_{z}^{\prime}\right]$

$w_{z}=-2 \rho\left(\xi \eta_{\rho}-\eta \xi_{\rho}\right)$

$=2 \rho\left[\lambda \eta_{\rho}^{\prime}\left(\lambda \xi^{\prime}+1\right)-\lambda^{2} \eta^{\prime} \xi_{\rho}^{\prime}\right]$.

$[1,3,6,7]$

\section{Derivation of Einstein- Maxwell Equations upto Fourth Order and Some Approximate} Solutions

To derive the EM equations upto fourth order and the solutions of aforesaid orders ; we substitute $f=h^{-1}$ and all its partial derivatives with respect to $\rho$ and $z$ into $(8 \mathrm{a}-\mathrm{d})$, we obtain $[1,3,6]$ :

$h \nabla^{2} h-h_{\rho}^{2}-h_{z}^{2}-\rho^{-2}\left(w_{\rho}^{2}+w_{z}^{2}\right)=-2 h^{3}\left(\Phi_{\rho}^{2}+\Phi_{z}^{2}+\Phi_{\rho}^{\prime 2}+\Phi_{z}^{\prime 2}\right)$,

$h \Delta w-2 h_{\rho} w_{\rho}-2 h_{z} w_{z}=4 \rho h^{3}\left(\Phi_{z}^{\prime} \Phi_{\rho}-\Phi_{\rho}^{\prime} \Phi_{z}\right)$,

$h \nabla^{2} \Phi^{\prime}=-h_{\rho} \Phi_{\rho}^{\prime}-h_{z} \Phi_{z}^{\prime}+\rho^{-1}\left(w_{\rho} \Phi_{z}-w_{z} \Phi_{\rho}\right)$,

$h \nabla^{2} \Phi=-h_{\rho} \Phi_{\rho}-h_{z} \Phi_{z}+\rho^{-1}\left(w_{z} \Phi_{\rho}^{\prime}-w_{\rho} \Phi_{z}^{\prime}\right)$.

Again we substitute $f=h^{-1}$ and all its partial derivatives with respect to $\rho$ and $z$ into (9a-b) and then after some manipulations we have [3] : 


$$
\begin{aligned}
& h^{2} \mu_{\rho}=h h_{\rho}+\frac{1}{2} \rho\left(h_{\rho}^{2}-h_{z}^{2}\right)+2 \rho h^{3}\left(\Phi_{z}^{2}-\Phi_{\rho}^{2}+\Phi_{z}^{\prime 2}-\Phi_{\rho}^{\prime 2}\right)+\frac{1}{2} \rho^{-1}\left(w_{z}^{2}-w_{\rho}^{2}\right), \\
& h^{2} \mu_{z}=h h_{z}+\rho h_{\rho} h_{z}-4 \rho h^{3}\left(\Phi_{\rho} \Phi_{z}+\Phi_{\rho}^{\prime} \Phi_{z}^{\prime}\right)-\rho^{-1} w_{\rho} w_{z} .
\end{aligned}
$$

We suppose that there is a solution of the field equations $(18 \mathrm{a}-\mathrm{d})$ which depends smoothly on the parameter $\lambda$ and a certain number of harmonic functions. The possible expansion of the functions $h, w, \Phi, \Phi^{\prime}$ in a power series in $\lambda$ are as follows [7] :

$h=\sum_{n=o}^{\infty} \lambda^{n} h^{(n)}, w=\sum_{n=o}^{\infty} \lambda^{n} w^{(n)}, \Phi=\sum_{n=o}^{\infty} \lambda^{n} \Phi^{(n)}, \Phi^{\prime}=\sum_{n=o}^{\infty} \lambda^{n} \Phi^{\prime(n)}$

We further suppose that the electromagnetic field vanishes and space- time becomes flat when $\lambda=0$, so that $h^{(0)}, w^{(0)}, \Phi^{(0)}, \Phi^{(0)}$ are constants, with $h^{(0)}=1, w^{(0)}=0$.

Making use of eq. (20) with their required partial derivatives into eqs. (18a - d) and by some manipulation we get equations up to $\lambda^{4}$ which respectively as follows [7]:

$$
\begin{aligned}
& \lambda \nabla^{2} h^{(1)}+\lambda^{2}\left[\nabla^{2} h^{(2)}+h^{(1)} \nabla^{2} h^{(1)}-\left(h_{\rho}^{(1) 2}+h_{z}^{(1) 2}\right)-\rho^{-2}\left(w_{\rho}^{(1) 2}+w_{z}^{(1) 2}\right)\right]+\lambda^{3}\left[\nabla^{2} h^{(3)}+h^{(1)} \nabla^{2} h^{(2)}\right. \\
& \left.+h^{(2)} \nabla^{2} h^{(1)}-2\left(h_{\rho}^{(1)} h_{\rho}^{(2)}+h_{z}^{(1)} h_{z}^{(2)}\right)-2 \rho^{-2}\left(w_{\rho}^{(1)} w_{\rho}^{(2)}+w_{z}^{(1)} w_{z}^{(2)}\right)\right] \\
& +\lambda^{4}\left[\nabla^{2} h^{(4)}+h^{(1)} \nabla^{2} h^{(3)}+h^{(2)} \nabla^{2} h^{(2)}+h^{(3)} \nabla^{2} h^{(1)}\right. \\
& -\left\{\left(h_{\rho}^{(2) 2}+h_{z}^{(2) 2}\right)+2\left(h_{\rho}^{(1)} h_{\rho}^{(3)}+h_{z}^{(1)} h_{z}^{(3)}\right)\right\} \\
& \left.-\rho^{-2}\left\{\left(w_{\rho}^{(2) 2}+w_{z}^{(2) 2}\right)+2\left(w_{\rho}^{(1)} w_{\rho}^{(3)}+w_{z}^{(1)} w_{z}^{(3)}\right)\right\}\right]+ \\
& =-2 \llbracket \lambda^{2}\left\{\Phi_{\rho}^{(1) 2}+\Phi_{z}^{(1) 2}+\Phi_{\rho}^{\prime(1) 2}+\Phi_{z}^{\prime(1) 2}\right\} \\
& +\lambda^{3}\left\{2\left(\Phi_{\rho}^{(1)} \Phi_{\rho}^{(2)}+\Phi_{z}^{(1)} \Phi_{z}^{(2)}+\Phi_{\rho}^{\prime(1)} \Phi_{\rho}^{\prime(2)}+\Phi_{z}^{\prime(1)} \Phi_{z}^{\prime(2)}\right)\right. \\
& \left.+3 h^{(1)}\left(\Phi_{\rho}^{(1) 2}+\Phi_{z}^{(1) 2}+\Phi_{\rho}^{\prime(1) 2}+\Phi_{z}^{\prime(1) 2}\right)\right\} \\
& +\lambda^{4}\left[\left\{1+3\left(h^{(2)}+h^{(1) 2}\right)\right\}\left\{\Phi_{\rho}^{(1) 2}+\Phi_{z}^{(1) 2}+\Phi_{\rho}^{\prime(1) 2}+\Phi_{z}^{\prime(1) 2}\right\}\right. \\
& \left.+\left(1+3 h^{(1)}\right)\left\{2\left(\Phi_{\rho}^{(1)} \Phi_{\rho}^{(2)}+\Phi_{z}^{(1)} \Phi_{z}^{(2)}+\Phi_{\rho}^{\prime(1)} \Phi_{\rho}^{\prime(2)}+\Phi_{z}^{\prime(1)} \Phi_{z}^{\prime(2)}\right)\right\}\right]+ \\
& \lambda \Delta w^{(1)}+\lambda^{2}\left\{\Delta w^{(2)}+h^{(1)} \Delta w^{(1)}-2\left(h_{\rho}^{(1)} w_{\rho}^{(1)}+h_{z}^{(1)} w_{z}^{(1)}\right)\right\} \\
& +\lambda^{3}\left[\Delta w^{(3)}+h^{(1)} \Delta w^{(2)}+h^{(2)} \Delta w^{(1)}-2\left\{\left(h_{\rho}^{(1)} w_{\rho}^{(2)}+h_{\rho}^{(2)} w_{\rho}^{(1)}\right)\right.\right. \\
& \left.\left.+\left(h_{z}^{(1)} w_{z}^{(2)}+h_{z}^{(2)} w_{z}^{(1)}\right)\right\}\right]+\lambda^{4}\left[\Delta w^{(4)}+h^{(1)} \Delta w^{(3)}+h^{(2)} \Delta w^{(2)}+h^{(3)} \Delta w^{(1)}\right. \\
& \left.-2\left\{\left(h_{\rho}^{(1)} w_{\rho}^{(3)}+h_{\rho}^{(2)} w_{\rho}^{(2)}+h_{\rho}^{(3)} w_{\rho}^{(1)}\right)+\left(h_{z}^{(1)} w_{z}^{(3)}+h_{z}^{(2)} w_{z}^{(2)}+h_{z}^{(3)} w_{z}^{(1)}\right)\right\}\right]+ \\
& =4 \rho \llbracket \lambda^{2}\left(\Phi_{z}^{\prime(1)} \Phi_{\rho}^{(1)}-\Phi_{\rho}^{\prime(1)} \Phi_{z}^{(1)}\right)+\lambda^{3}\left[\left(\Phi_{z}^{\prime(1)} \Phi_{\rho}^{(2)}+\Phi_{z}^{\prime(2)} \Phi_{\rho}^{(1)}\right)\right. \\
& \left.-\left(\Phi_{\rho}^{\prime(1)} \Phi_{z}^{(2)}+\Phi_{\rho}^{\prime(2)} \Phi_{z}^{(1)}\right)+3 h^{(1)}\left(\Phi_{z}^{\prime(1)} \Phi_{\rho}^{(1)}-\Phi_{\rho}^{\prime(1)} \Phi_{z}^{(1)}\right)\right] \\
& +\lambda^{4}\left[\left(\Phi_{z}^{\prime(1)} \Phi_{\rho}^{(3)}+\Phi_{z}^{\prime(2)} \Phi_{\rho}^{(2)}+\Phi_{z}^{\prime(3)} \Phi_{\rho}^{(1)}\right)-\left(\Phi_{\rho}^{\prime(1)} \Phi_{z}^{(3)}+\Phi_{\rho}^{\prime(2)} \Phi_{z}^{(2)}+\Phi_{\rho}^{\prime(3)} \Phi_{z}^{(1)}\right)\right. \\
& +3 h^{(1)}\left\{\left(\Phi_{z}^{\prime(1)} \Phi_{\rho}^{(2)}+\Phi_{z}^{(2)} \Phi_{\rho}^{(1)}\right)-\left(\Phi_{\rho}^{\prime(1)} \Phi_{z}^{(2)}+\Phi_{\rho}^{\prime(2)} \Phi_{z}^{(1)}\right)\right\} \\
& \left.\left.+\left(3 h^{(2)}+3 h^{(1) 2}\right)\left(\Phi_{z}^{\prime(1)} \Phi_{\rho}^{(1)}-\Phi_{\rho}^{\prime(1)} \Phi_{z}^{(1)}\right)\right]+\ldots . . . . .1\right] . \\
& \lambda \nabla^{2} \Phi^{\prime(1)}+\lambda^{2}\left(\nabla^{2} \Phi^{\prime(2)}+h^{(1)} \nabla^{2} \Phi^{\prime(1)}\right)+\lambda^{3}\left(\nabla^{2} \Phi^{\prime(3)}+h^{(1)} \nabla^{2} \Phi^{\prime(2)}+h^{(2)} \nabla^{2} \Phi^{\prime(1)}\right) \\
& +\lambda^{4}\left(\nabla^{2} \Phi^{\prime(4)}+h^{(1)} \nabla^{2} \Phi^{\prime(3)}+h^{(2)} \nabla^{2} \Phi^{\prime(2)}+h^{(3)} \nabla^{2} \Phi^{(1)}\right)+\ldots \ldots \ldots \ldots . . . \\
& =-\left[\lambda^{2}\left\{\left(h_{\rho}^{(1)} \Phi_{\rho}^{(1)}+h_{z}^{(1)} \Phi_{z}^{(1)}\right)-\rho^{-1}\left(w_{\rho}^{(1)} \Phi_{z}^{(1)}-w_{z}^{(1)} \Phi_{\rho}^{(1)}\right)\right\}\right. \\
& +\lambda^{3}\left\{\left(h_{\rho}^{(1)} \Phi_{\rho}^{\prime(2)}+h_{\rho}^{(2)} \Phi_{\rho}^{\prime(1)}+h_{z}^{(1)} \Phi_{z}^{\prime(2)}+h_{z}^{(2)} \Phi_{z}^{\prime(1)}\right)\right. \\
& \left.-\rho^{-1}\left(w_{\rho}^{(1)} \Phi_{z}^{(2)}+w_{\rho}^{(2)} \Phi_{z}^{(1)}-w_{z}^{(1)} \Phi_{\rho}^{(2)}-w_{z}^{(2)} \Phi_{\rho}^{(1)}\right)\right\}
\end{aligned}
$$


$+\lambda^{4}\left\{\left(h_{\rho}^{(1)} \Phi_{\rho}^{\prime(3)}+h_{\rho}^{(2)} \Phi_{\rho}^{(2)}+h_{\rho}^{(3)} \Phi_{\rho}^{\prime(1)}+h_{z}^{(1)} \Phi_{z}^{\prime(3)}+h_{z}^{(2)} \Phi_{z}^{(2)}+h_{z}^{(3)} \Phi_{z}^{\prime(1)}\right)\right.$
$\left.\left.-\rho^{-1}\left(w_{\rho}^{(1)} \Phi_{z}^{(3)}+w_{\rho}^{(2)} \Phi_{z}^{(2)}+w_{\rho}^{(3)} \Phi_{z}^{(1)}-w_{z}^{(1)} \Phi_{\rho}^{(3)}-w_{z}^{(2)} \Phi_{\rho}^{(2)}-w_{z}^{(3)} \Phi_{\rho}^{(1)}\right)\right\}\right]+\ldots \ldots \ldots .$.

$\lambda \nabla^{2} \Phi^{(1)}+\lambda^{2}\left(\nabla^{2} \Phi^{(2)}+h^{(1)} \nabla^{2} \Phi^{(1)}\right)+\lambda^{3}\left(\nabla^{2} \Phi^{(3)}+h^{(1)} \nabla^{2} \Phi^{(2)}+h^{(2)} \nabla^{2} \Phi^{(1)}\right)$

$+\lambda^{4}\left(\nabla^{2} \Phi^{(4)}+h^{(1)} \nabla^{2} \Phi^{(3)}+h^{(2)} \nabla^{2} \Phi^{(2)}+h^{(3)} \nabla^{2} \Phi^{(1)}\right)+$

$=-\left[\lambda^{2}\left\{\left(h_{\rho}^{(1)} \Phi_{\rho}^{(1)}+h_{z}^{(1)} \Phi_{z}^{(1)}\right)-\rho^{-1}\left(w_{z}^{(1)} \Phi_{\rho}^{\prime(1)}-w_{\rho}^{(1)} \Phi_{z}^{\prime(1)}\right)\right\}\right.$

$+\lambda^{3}\left\{\left(h_{\rho}^{(1)} \Phi_{\rho}^{(2)}+h_{\rho}^{(2)} \Phi_{\rho}^{(1)}+h_{z}^{(1)} \Phi_{z}^{(2)}+h_{z}^{(2)} \Phi_{z}^{(1)}\right)-\rho^{-1}\left(w_{z}^{(1)} \Phi_{\rho}^{\prime(2)}+w_{z}^{(2)} \Phi_{\rho}^{\prime(1)}-w_{\rho}^{(1)} \Phi_{z}^{\prime(2)}-w_{\rho}^{(2)} \Phi_{z}^{\prime(1)}\right)\right\}$

$+\lambda^{4}\left\{\left(h_{\rho}^{(1)} \Phi_{\rho}^{(3)}+h_{\rho}^{(2)} \Phi_{\rho}^{(2)}+h_{\rho}^{(3)} \Phi_{\rho}^{(1)}+h_{z}^{(1)} \Phi_{z}^{(3)}+h_{z}^{(2)} \Phi_{z}^{(2)}+h_{z}^{(3)} \Phi_{Z}^{(1)}\right)\right.$

$\left.\left.-\rho^{-1}\left(w_{z}^{(1)} \Phi_{\rho}^{(3)}+w_{z}^{(2)} \Phi_{\rho}^{(2)}+w_{z}^{(3)} \Phi_{\rho}^{(1)}-w_{\rho}^{(1)} \Phi_{z}^{\prime(3)}-w_{\rho}^{(2)} \Phi_{z}^{\prime(2)}-w_{\rho}^{(3)} \Phi_{z}^{\prime(1)}\right)\right\}\right]+$

Equating the co-efficients of $\lambda$, that is first order from $(21 \mathrm{a}-\mathrm{d})$, we get respectively :

$\nabla^{2} h^{(1)}=0, \Delta w^{(1)}=0, \nabla^{2} \Phi^{(1)}=0, \nabla^{2} \Phi^{(1)}=0$.

The solution of first and last two equations of (22) are respectively

$h^{(1)}=\tau, \Phi^{(1)}=\xi, \Phi^{\prime(1)}=\eta$,

where $\tau, \xi, \eta$ are independent harmonic functions [1,3,5]. The proof is obvious.

The second equation of (22) can be solved as follows :

$$
w_{\rho}^{(1)}=\rho \sigma_{z}, w_{z}^{(1)}=-\rho \sigma_{\rho},
$$

where $\sigma$ is a harmonic function.

If $\zeta$ is harmonic, then (24) is equivalent to the solution

$$
w^{(1)}=\rho \zeta_{\rho},
$$

which is used in (1.11a) (Salam, 2010) [7], but (25) preferable for the present. In fact $\sigma=-\zeta_{z}$

In the second order, that is equating the co-efficients of $\lambda^{2}$ from both sides of $(21 \mathrm{a}-\mathrm{d})$ and using (22), we get respectively

$$
\begin{aligned}
& \nabla^{2} h^{(2)}-\left(h_{\rho}^{(1) 2}+h_{z}^{(1) 2}\right)-\rho^{-2}\left(w_{\rho}^{(1) 2}+w_{z}^{(1) 2}\right)=-2\left\{\Phi_{\rho}^{(1) 2}+\Phi_{z}^{(1) 2}+\Phi_{\rho}^{\prime(1) 2}+\Phi_{z}^{\prime(1) 2}\right\}, \\
& \Delta w^{(2)}-2\left(h_{\rho}^{(1)} w_{\rho}^{(1)}+h_{z}^{(1)} w_{z}^{(1)}\right)=4 \rho\left(\Phi_{z}^{\prime(1)} \Phi_{\rho}^{(1)}-\Phi_{\rho}^{\prime(1)} \Phi_{z}^{(1)}\right), \\
& \nabla^{2} \Phi^{\prime(2)}=-\left[\left(h_{\rho}^{(1)} \Phi_{\rho}^{\prime(1)}+h_{z}^{(1)} \Phi_{z}^{(1)}\right)-\rho^{-1}\left(w_{\rho}^{(1)} \Phi_{z}^{(1)}-w_{z}^{(1)} \Phi_{\rho}^{(1)}\right)\right] \\
& \nabla^{2} \Phi^{(2)}=-\left[\left(h_{\rho}^{(1)} \Phi_{\rho}^{(1)}+h_{z}^{(1)} \Phi_{z}^{(1)}\right)-\rho^{-1}\left(w_{z}^{(1)} \Phi_{\rho}^{\prime(1)}-w_{\rho}^{(1)} \Phi_{z}^{\prime(1)}\right)\right] .
\end{aligned}
$$

Substituting the values of (23) and (24) into (26a-d) yields respectively the following equations [7] :

$\nabla^{2} h^{(2)}=\tau_{\rho}^{2}+\tau_{z}^{2}+\sigma_{\rho}^{2}+\sigma_{z}^{2}-2\left(\xi_{\rho}^{2}+\xi_{z}^{2}+\eta_{\rho}^{2}+\eta_{z}^{2}\right)$,

$\Delta w^{(2)}=2 \rho\left(\tau_{\rho} \sigma_{z}-\tau_{z} \sigma_{\rho}\right)+4 \rho\left(\eta_{z} \xi_{\rho}-\eta_{\rho} \xi_{z}\right)$,

$\nabla^{2} \Phi^{\prime(2)}=-\tau_{\rho} \eta_{\rho}-\tau_{z} \eta_{z}+\xi_{\rho} \sigma_{\rho}+\xi_{z} \sigma_{z}$,

$\nabla^{2} \Phi^{(2)}=-\tau_{\rho} \xi_{\rho}-\tau_{z} \xi_{z}-\eta_{\rho} \sigma_{\rho}-\eta_{z} \sigma_{z} \cdot$

The solutions of $(27 \mathrm{a}-\mathrm{d})$ can be taken as follows :

$$
\left.\begin{array}{l}
h^{(2)}=\frac{1}{2} \tau^{2}+\frac{1}{2} \sigma^{2}-\xi^{2}-\eta^{2}, \\
w_{\rho}^{(2)}=\rho\left(\tau \sigma_{z}-\sigma \tau_{z}\right)+2 \rho\left(\xi \eta_{z}-\eta \xi_{z}\right) \\
w_{z}^{(2)}=-\rho\left(\tau \sigma_{\rho}-\sigma \tau_{\rho}\right)-2 \rho\left(\xi \eta_{\rho}-\eta \xi_{\rho}\right) \\
\Phi^{(2)}=-\frac{1}{2} \tau \eta+\frac{1}{2} \xi \sigma,
\end{array}\right\},
$$


$\Phi^{(2)}=-\frac{1}{2} \tau \xi-\frac{1}{2} \eta \sigma$

The consistency of (28b) is guaranteed by the fact that $\tau, \sigma, \xi, \eta$ are harmonic functions. We have ignored arbitrary harmonic functions that could be added to $(28 \mathrm{a}, \mathrm{c}, \mathrm{d})$ and an arbitrary solution of $\Delta w^{(2)}=0$ that could be added to $w^{(2)}$. Equations (27a,c,d) can be solved by repeated application of the identity.

$\nabla^{2}(G H)=G \nabla^{2} H+H \nabla^{2} G+2\left(G_{\rho} H_{\rho}+G_{z} H_{z}\right)$,

where $G, H$ are any two functions of $\rho$,z. The equation (27b) has been solved in a similar way to which

$\Delta w^{(2)}=2\left(1-\beta^{2}\right)^{\frac{1}{2}} \rho\left(\sigma_{z} \zeta_{\rho z}-\sigma_{\rho} \zeta_{z z}\right)$.

was solved [7].

Equating the co-efficients of $\lambda^{3}$ i.e, the third order from (21a-d), we get, using (22),

$\nabla^{2} h^{(3)}+h^{(1)} \nabla^{2} h^{(2)}-2\left(h_{\rho}^{(1)} h_{\rho}^{(2)}+h_{z}^{(1)} h_{z}^{(2)}\right)-2 \rho^{-2}\left(w_{\rho}^{(1)} w_{\rho}^{(2)}+w_{z}^{(1)} w_{z}^{(2)}\right)=-2\left[2\left(\Phi_{\rho}^{(1)} \Phi_{\rho}^{(2)}\right.\right.$

$\left.\left.+\Phi_{z}^{(1)} \Phi_{z}^{(2)}+\Phi_{\rho}^{\prime(1)} \Phi_{\rho}^{\prime(2)}+\Phi_{z}^{(1)} \Phi_{z}^{(2)}\right)+3 h^{(1)}\left(\Phi_{\rho}^{(1) 2}+\Phi_{z}^{(1) 2}+\Phi_{\rho}^{\prime(1) 2}+\Phi_{z}^{\prime(1) 2}\right)\right]$

$\Delta w^{(3)}+h^{(1)} \Delta w^{(2)}-2\left\{\left(h_{\rho}^{(1)} w_{\rho}^{(2)}+h_{\rho}^{(2)} w_{\rho}^{(1)}\right)+\left(h_{z}^{(1)} w_{z}^{(2)}+h_{z}^{(2)} w_{z}^{(1)}\right)\right\}=4 \rho\left[\left(\Phi_{z}^{\prime(1)} \Phi_{\rho}^{(2)}+\Phi_{z}^{\prime(2)} \Phi_{\rho}^{(1)}\right)\right.$

$\left.-\left(\Phi_{\rho}^{\prime(1)} \Phi_{z}^{(2)}+\Phi_{\rho}^{(2)} \Phi_{z}^{(1)}\right)+3 h^{(1)}\left(\Phi_{z}^{\prime(1)} \Phi_{\rho}^{(1)}-\Phi_{\rho}^{\prime(1)} \Phi_{z}^{(1)}\right)\right]$

$\nabla^{2} \Phi^{\prime(3)}+h^{(1)} \nabla^{2} \Phi^{\prime(2)}=-\left[\left(h_{\rho}^{(1)} \Phi_{\rho}^{\prime(2)}+h_{\rho}^{(2)} \Phi_{\rho}^{\prime(1)}+h_{z}^{(1)} \Phi_{z}^{\prime(2)}+h_{z}^{(2)} \Phi_{z}^{(1)}\right)-\rho^{-1}\left(w_{\rho}^{(1)} \Phi_{z}^{(2)}+w_{\rho}^{(2)} \Phi_{z}^{(1)}\right.\right.$

$\left.\left.-w_{z}^{(1)} \Phi_{\rho}^{(2)}-w_{z}^{(2)} \Phi_{\rho}^{(1)}\right)\right]$

$\nabla^{2} \Phi^{(3)}+h^{(1)} \nabla^{2} \Phi^{(2)}=-\left[\left(h_{\rho}^{(1)} \Phi_{\rho}^{(2)}+h_{\rho}^{(2)} \Phi_{\rho}^{(1)}+h_{z}^{(1)} \Phi_{z}^{(2)}+h_{z}^{(2)} \Phi_{z}^{(1)}\right)-\rho^{-1}\left(w_{z}^{(1)} \Phi_{\rho}^{(2)}+w_{z}^{(2)} \Phi_{\rho}^{\prime(1)}\right.\right.$ $\left.\left.-w_{\rho}^{(1)} \Phi_{z}^{\prime(2)}-w_{\rho}^{(2)} \Phi_{z}^{\prime(1)}\right)\right]$.

Using equations (23), (24), (28a $-\mathrm{d})$ in the aforesaid equations $(31 \mathrm{a}-\mathrm{d})$ and then by some manipulations, we obtain

$$
\begin{aligned}
& \nabla^{2} h^{(3)}=\tau\left(\tau_{\rho}^{2}+\tau_{z}^{2}\right)+\tau\left(\sigma_{\rho}^{2}+\sigma_{z}^{2}\right)-2 \tau\left(\xi_{\rho}^{2}+\xi_{z}^{2}+\eta_{\rho}^{2}+\eta_{z}^{2}\right)-2 \xi\left(\tau_{\rho} \xi_{\rho}+\tau_{z} \xi_{z}\right) \\
& -2 \eta\left(\tau_{\rho} \eta_{\rho}+\tau_{z} \eta_{z}\right)+2 \xi\left(\sigma_{\rho} \eta_{\rho}+\sigma_{z} \eta_{z}\right)-2 \eta\left(\sigma_{\rho} \xi_{\rho}+\sigma_{z} \xi_{z}\right), \\
& \Delta w^{(3)}=2 \rho\left[2 \tau\left(\xi_{\rho} \eta_{z}-\xi_{z} \eta_{\rho}\right)+\tau\left(\tau_{\rho} \sigma_{z}-\tau_{z} \sigma_{\rho}\right)+\tau_{\rho}\left(\xi \eta_{z}-\xi_{z} \eta\right)-\tau_{z}\left(\xi \eta_{\rho}-\xi_{\rho} \eta\right)\right. \\
& \left.-\sigma_{z}\left(\xi \xi_{\rho}+\eta \eta_{\rho}\right)+\sigma_{\rho}\left(\xi \xi_{z}+\eta \eta_{z}\right)\right] \\
& \nabla^{2} \Phi^{\prime(3)}=\frac{1}{2} \eta\left(\tau_{\rho}^{2}+\tau_{\mathrm{z}}^{2}\right)+\frac{1}{2} \tau\left(\tau_{\rho} \eta_{\rho}+\tau_{\mathrm{z}} \eta_{\mathrm{z}}\right)-\xi\left(\tau_{\rho} \sigma_{\rho}+\tau_{\mathrm{z}} \sigma_{\mathrm{z}}\right)-\frac{3}{2} \sigma\left(\tau_{\rho} \xi_{\rho}+\tau_{\mathrm{z}} \xi_{\mathrm{z}}\right) \\
& -\frac{3}{2} \sigma\left(\sigma_{\rho} \eta_{\rho}+\sigma_{\mathrm{z}} \eta_{\mathrm{z}}\right)+2 \eta\left(\eta_{\rho}^{2}+\eta_{\mathrm{z}}^{2}\right)+4 \xi\left(\xi_{\rho} \eta_{\rho}+\xi_{\mathrm{z}} \eta_{\mathrm{z}}\right)-2 \eta\left(\xi_{\rho}^{2}+\xi_{\mathrm{z}}^{2}\right)-\frac{1}{2} \tau\left(\sigma_{\rho} \xi_{\rho}+\sigma_{\mathrm{z}} \xi_{\mathrm{z}}\right) \\
& -\frac{1}{2} \eta\left(\sigma_{\rho}^{2}+\sigma_{\mathrm{z}}^{2}\right)
\end{aligned}
$$

$\nabla^{2} \Phi^{(3)}=\frac{1}{2} \xi\left(\tau_{\rho}^{2}+\tau_{\mathrm{z}}^{2}\right)+\frac{1}{2} \tau\left(\tau_{\rho} \xi_{\rho}+\tau_{\mathrm{z}} \xi_{\mathrm{z}}\right)+\eta\left(\tau_{\rho} \sigma_{\rho}+\tau_{\mathrm{z}} \sigma_{\mathrm{z}}\right)+\frac{3}{2} \sigma\left(\tau_{\rho} \eta_{\rho}+\tau_{\mathrm{z}} \eta_{\mathrm{z}}\right)$

$-\frac{3}{2} \sigma\left(\sigma_{\rho} \xi_{\rho}+\sigma_{\mathrm{z}} \xi_{\mathrm{z}}\right)+2 \xi\left(\xi_{\rho}^{2}+\xi_{\mathrm{z}}^{2}\right)+4 \eta\left(\xi_{\rho} \eta_{\rho}+\xi_{\mathrm{z}} \eta_{\mathrm{z}}\right)-2 \xi\left(\eta_{\rho}^{2}+\eta_{\mathrm{z}}^{2}\right)+\frac{1}{2} \tau\left(\eta_{\rho} \sigma_{\rho}+\eta_{\mathrm{z}} \sigma_{\mathrm{z}}\right)$

$-\frac{1}{2} \xi\left(\sigma_{\rho}^{2}+\sigma_{\mathrm{z}}^{2}\right)$. 
So far we have not succeeded in finding closed form solutions of any of (32a-d) in terms of the harmonic functions $\tau, \sigma, \xi, \eta$. It is possible that such solutions do not exist. At each state of this approximation scheme one gets a Poisson equation for $h^{(\mathrm{n})}$ etc. , in which the right-hand side is given in terms of the lower-order functions, which are known in principle [2,3,6]. The equations for the $w^{(\mathrm{n})}$ can be converted into Poisson's equations as follows: Let the equation at the nth stage be

$\Delta w^{(\mathrm{n})}=F(\rho, \mathrm{z})$,

where $F$ is known in terms of lower-order functions. Define $w^{(\mathrm{n})}$ by $w^{(\mathrm{n})}=\rho w_{\rho}^{(\mathrm{n})}$; it is then easy to verify the identity given by the first of the forthcoming equations :

$\Delta w^{(\mathrm{n})}=\rho\left(\nabla^{2} w^{(\mathrm{n})}\right)_{\rho}, \nabla^{2} w^{(\mathrm{n})}=\int \frac{\rho}{\rho^{\prime}} F\left(\rho^{\prime}, \mathrm{z}\right) d \rho^{\prime}$,

the second equation being the resulting Poisson's equation. These equations can be solved at each stage in terms of integral representations by the standard methods (Islam (1985), Chapter 1) [6], but such a procedure is not very useful.

Equations (32a-d) can be considered as a "laboratory" because with these equations we can test the possibility of exact solutions in terms of harmonic functions. The following identity is useful for these purpose $[1,3,6]$ :

$$
\begin{aligned}
\nabla^{2}(F G H) & =F G \nabla^{2} H+F H \nabla^{2} G+G H \nabla^{2} F+2 F\left(H_{\rho} G_{\rho}+H_{z} G_{z}\right)+2 H\left(F_{\rho} G_{\rho}+F_{z} G_{z}\right) \\
+ & 2 G\left(H_{\rho} F_{\rho}+H_{z} F_{z}\right),
\end{aligned}
$$

for any three functions $F, G$ and $H$.

\section{Physical Interpretation}

When $\lambda=0$, the space-time becomes flat and the fields vanish $[1,3,6]$. Thus the approximate solution represents a weak field in some sense. The constant $\lambda$ can be taken to be proportional to the gravitational constant and charge of the sources. If $\lambda$ is zero the gravitational constant vanishes so that the space is flat and the charges giving rise to the electromagnetic field vanish so that the fields are zero. For convenience we consider the functions upto $\lambda^{2}$ terms only. To examine the possible sources for these solutions, we choose the harmonic functions such a way that the space-time is asymptotically flat to this order (Islam (1985), Chapter 1) [6]. To this end let

$$
\tau=\tau^{\prime}+k_{1} \xi+k_{2} \eta
$$

where $k_{1}, k_{2}$ are constants (which are different from earlier constants), $\tau^{\prime}$ is a harmonic function and $\tau^{\prime}, \sigma, \xi, \eta$ have the following forms at infinity :

$$
\begin{aligned}
& \tau^{\prime}=A_{1} r^{-1}+A_{1}^{\prime} z r^{-3}+\ldots \ldots \ldots, \sigma=A_{2} z r^{-3}+A_{2}^{\prime}\left(r^{-3}-3 z^{2} r^{-5}\right)+\ldots \ldots \ldots \\
& \xi=B_{1} r^{-1}+B_{1}^{\prime} z r^{-3}+\ldots \ldots \ldots, \eta \ldots, \quad \eta=B_{2} r^{-1}+B_{2}^{\prime} z r^{-3}+\ldots \ldots \ldots \ldots \ldots .
\end{aligned}
$$

where $r^{2}=\left(\rho^{2}+\mathrm{z}^{2}\right)$, and the dots represent terms which vanish at infinity faster than the preceding terms. The reason for choosing the leading term for $\sigma$ as in (37) is that this leads to the correct asymptotic behaviour for $w$, which must tend to zero at infinity like $\rho^{2} r^{-3}$ (Misner et al (1973), Chapter 19) [16]. The behaviour of the functions upto $\lambda^{2}$ terms and $r^{-2}$ terms is then as follows [1,3,6]:

$$
\begin{aligned}
h= & 1+\lambda\left[\left(A_{1}+k_{1} B_{1}+k_{2} B_{2}\right) r^{-1}+\left(A_{1}^{\prime}+k_{1} B_{1}^{\prime}+k_{2} B_{2}^{\prime}\right) z r^{-3}\right] \\
& \left.+\lambda^{2}\left[\frac{1}{2}\left(A_{1}+k_{1} B_{1}+k_{2} B_{2}\right)^{2}-B_{1}^{2}-B_{2}^{2}\right)\right] r^{-2}, \\
w= & -\lambda\left(A_{2} \rho^{2} r^{-3}-3 A_{2}^{\prime} \rho^{2} z r^{-5}\right)+\lambda^{2} A^{\prime} \rho^{2} r^{-4} \\
A^{\prime} \equiv & -\frac{1}{2} A_{2}\left(A_{1}+k_{1} B_{1}+k_{2} B_{2}\right)+\left(B_{2} B_{1}^{\prime}-B_{2}^{\prime} B_{1}\right), \\
\Phi= & 1+\lambda\left(B_{1} r^{-1}+B_{1}^{\prime} z r^{-3}\right)-\frac{1}{2} \lambda^{2} B_{1}\left(A_{1}+k_{1} B_{1}+k_{2} B_{2}\right) r^{-2},
\end{aligned}
$$




$$
\Phi^{\prime}=\lambda\left(B_{2} r^{-1}+B_{2}^{\prime} z r^{-3}\right)-\frac{1}{2} \lambda^{2} B_{2}\left(A_{1}+k_{1} B_{1}+k_{2} B_{2}\right) r^{-2}
$$

The forgoing equations represent fairly general asymptotic behaviour. For example, one can have the correct asymptotic behaviour for $w$ given by (38b) and yet have a non-zero mass, unlike the Bonnor [14] or Papapetrou [9] solutions. For the Bonnor solutions, we let [3],

$$
(\tau, \sigma, \xi, \eta)=\left(a_{1} \sigma^{\prime}, a_{2} \sigma^{\prime}, a_{3} \sigma^{\prime}, a_{4} \sigma^{\prime}\right) \text {, }
$$

where $a_{i}$ are constants implies that $\sigma$ is proportional to $\tau$ which means that if $\sigma$ is to have the asymptotic behaviour as in (37), from (36), (37), we have

$$
A_{1}+k_{1} B_{1}+k_{2} B_{2}=0
$$

which implies that the mass of the source is zero. Similarly from the conditions (Salam (1988), eq. (3.20)) [3]

$$
(\tau, \sigma, \xi, \eta)=\left(2 \xi^{\prime},-2 \eta^{\prime},-\xi^{\prime},-\eta^{\prime}\right)
$$

for the PIW solutions imply the following relations :

$$
A_{1}+k_{1} B_{1}+k_{2} B_{2}=-2 B_{1}, \quad B_{2}=0,2 B_{2}^{\prime}=A_{2} .
$$

These relations imply that the corresponding sources satisfy (1), since for example $\lambda B_{2}^{\prime}$ and $\frac{1}{2} \lambda A_{2}$ are related to the magnetic moment and angular momentum respectively. In equation (42), $\frac{1}{2} \lambda\left(A_{1}+k_{1} B_{1}+k_{2} B_{2}\right)$ and $\lambda B_{1}$ represent the mass and charge, so that the mass is equal to the charge. With the help of these techniques one can show that the sources for the class of the forgoing solutions satisfy (2). In the absence of the magnetic monopoles, we should have $B_{2}=0$ [7].

\section{Higher Order Approximate Solutions Of EM Equations}

In section (IV), we obtained approximate solutions of EM equations in terms of four harmonic functions $\sigma, \tau, \xi, \eta$. In that section we have shown the solutions upto the second order. The third order explicit solutions in closed form in terms of the above harmonic functions has been obtained by Salam $(1988,2000)$ $[3,1]$. But the fourth order equations could not be solved in closed form. In this section firstly we obtain the fourth order equations in terms of the harmonic functions $\sigma, \tau, \xi, \eta$ and then we shall find their explicit solutions for the particular choice of the harmonic functions.

Before deriving the fourth order equations and finding their solutions, we briefly describe the procedures for the solutions of third order equations and mention their solutions. Making use of equations (36) and (37) and their partial derivatives with respect to $\rho$ and $z$ into (32a-d), and then by some manipulations and keeping only upto $r^{-5}$ terms, we have the following respectively [1,3]:

$\nabla^{2} h^{(3)}=\alpha_{1} r^{-5}+$

where

$$
\begin{gathered}
\alpha_{1}=\left(A_{1}+k_{1} B_{1}+k_{2} B_{2}\right)\left[A_{1}^{2}+k_{1}^{2} B_{1}^{2}+k_{2}^{2} B_{2}^{2}+2\left(k_{1} A_{1} B_{1}+k_{2} A_{1} B_{2}+k_{1} k_{2} B_{1} B_{2}\right)\right. \\
\left.+4\left(B_{1}^{2}+B_{2}^{2}\right)\right] .
\end{gathered}
$$

The solution of (43) can be taken as $h^{(3)}=\beta_{1} r^{-3}$.

Now to find out the value of $\beta_{1}$, applying $\nabla^{2}$ on both sides of (44) and then by some manipulations and comparing with (43), we obtain

$$
\beta_{1}=\frac{1}{6} \alpha_{1}
$$

Substituting the value of $\beta_{1}$ into (44), we get the required solution of (43) as

$$
h^{(3)}=\frac{1}{6} \alpha_{1} r^{-3} \text {. }
$$

From (32b), we have

$$
\Delta w^{(3)}=\alpha_{2} \rho^{2} r^{-7}+
$$

where $\alpha_{2}=\left\{6\left(A_{1}+k_{1} B_{1}+k_{2} B_{2}\right)\left(B_{1}^{\prime} B_{2}-B_{1} B_{2}^{\prime}\right)+2 A_{2}\left(B_{1}^{2}+B_{1}^{2}\right)-2\left(A_{1}+k_{1} B_{1}+k_{2} B_{2}\right)^{2} A_{2}\right\}$. 
The solution of (47) can be taken as

$$
w^{(3)}=\beta_{2} \rho^{2} r^{-5}
$$

Applying $\Delta$ on both sides of (48) and then by some simplifications and comparing with (47), we get

$$
\beta_{2}=\frac{1}{10} \alpha_{2} \text {. }
$$

Putting the value of $\beta_{2}$ into (48), we get the required solution of (47):

$$
w^{(3)}=\frac{1}{10} \alpha_{2} \rho^{2} r^{-5} \text {. }
$$

From (32c), we have

$$
\nabla^{2} \Phi^{\prime(3)}=\alpha_{3} r^{-5}+
$$

where $\alpha_{3}=B_{2}\left(A_{1}+k_{1} B_{1}+k_{2} B_{2}\right)^{2}+4 B_{1}^{2} B_{2}$.

The solution of (51) can be taken as

$$
\Phi^{\prime(3)}=\beta_{3} r^{-3}
$$

Following the foregoing technique of (45), yields

$$
\beta_{3}=\frac{1}{6} \alpha_{3} \text {. }
$$

Hence the solution of (51) becomes

$$
\Phi^{\prime(3)}=\frac{1}{6} \alpha_{3} r^{-3} \text {. }
$$

Finally from (32d), we obtain

$$
\nabla^{2} \Phi^{(3)}=\alpha_{4} r^{-5}+\ldots \ldots \ldots \ldots \ldots \ldots \ldots \ldots,
$$

where $\alpha_{4}=B_{1}\left\{\left(A_{1}+k_{1} B_{1}+k_{2} B_{2}\right)^{2}+2\left(B_{1}^{2}+B_{2}^{2}\right)\right\}$.

The solution of (55) may be taken as

$$
\Phi^{(3)}=\beta_{4} r^{-3} \text {. }
$$

Following the similar method of (45), we have

$$
\beta_{4}=\frac{1}{6} \alpha_{4} \text {. }
$$

Substituting the value of $\beta_{4}$ into (56), we obtain the required solution of (55) is

$$
\Phi^{(3)}=\frac{1}{6} \alpha_{4} r^{-3} \text {. }
$$

In the fourth order, that is equating the co-efficients of $\lambda^{4}$ from both sides of (21a-d) and making use of (22), we get respectively [7]

$$
\begin{aligned}
& \nabla^{2} h^{(4)}+h^{(1)} \nabla^{2} h^{(3)}+h^{(2)} \nabla^{2} h^{(2)}-\left\{\left(h_{\rho}^{(2) 2}+h_{z}^{(2) 2}\right)+2\left(h_{\rho}^{(1)} h_{\rho}^{(3)}+h_{z}^{(1)} h_{z}^{(3)}\right)\right\} \\
& -\rho^{-2}\left\{\left(w_{\rho}^{(2) 2}+w_{z}^{(2) 2}\right)+2\left(w_{\rho}^{(1)} w_{\rho}^{(3)}+w_{z}^{(1)} w_{z}^{(3)}\right)\right\} \\
& =-2\left[\left\{1+3\left(h^{(2)}+h^{(1) 2}\right)\right\}\left\{\Phi_{\rho}^{(1) 2}+\Phi_{z}^{(1) 2}+\Phi_{\rho}^{(1) 2}+\Phi_{z}^{(1) 2}\right\}\right. \\
& \left.+\left(1+3 h^{(1)}\right)\left\{2\left(\Phi_{\rho}^{(1)} \Phi_{\rho}^{(2)}+\Phi_{z}^{(1)} \Phi_{z}^{(2)}+\Phi_{\rho}^{\prime(1)} \Phi_{\rho}^{\prime(2)}+\Phi_{z}^{\prime(1)} \Phi_{z}^{\prime(2)}\right)\right\}\right], \\
& \Delta w^{(4)}+h^{(1)} \Delta w^{(3)}+h^{(2)} \Delta w^{(2)}-2\left\{\left(h_{\rho}^{(1)} w_{\rho}^{(3)}+h_{\rho}^{(2)} w_{\rho}^{(2)}+h_{\rho}^{(3)} w_{\rho}^{(1)}\right)\right. \\
& \left.+\left(h_{z}^{(1)} w_{z}^{(3)}+h_{z}^{(2)} w_{z}^{(2)}+h_{z}^{(3)} w_{z}^{(1)}\right)\right\} \\
& =4 \rho\left[\left(\Phi_{z}^{\prime(1)} \Phi_{\rho}^{(3)}+\Phi_{z}^{(2)} \Phi_{\rho}^{(2)}+\Phi_{z}^{\prime(3)} \Phi_{\rho}^{(1)}\right)-\left(\Phi_{\rho}^{(1)} \Phi_{z}^{(3)}+\Phi_{p}^{(2)} \Phi_{z}^{(2)}+\Phi_{\rho}^{\prime(3)} \Phi_{z}^{(1)}\right)\right. \\
& +3 h^{(1)}\left\{\left(\Phi_{z}^{\prime(1)} \Phi_{\rho}^{(2)}+\Phi_{z}^{\prime(2)} \Phi_{\rho}^{(1)}\right)-\left(\Phi_{\rho}^{\prime(1)} \Phi_{z}^{(2)}+\Phi_{\rho}^{\prime(2)} \Phi_{z}^{(1)}\right)\right\}
\end{aligned}
$$


$\left.+\left(3 h^{(2)}+3 h^{(1) 2}\right)\left(\Phi_{z}^{(1)} \Phi_{\rho}^{(1)}-\Phi_{\rho}^{\prime(1)} \Phi_{z}^{(1)}\right)\right]$,

$\nabla^{2} \Phi^{\prime(4)}+h^{(1)} \nabla^{2} \Phi^{\prime(3)}+h^{(2)} \nabla^{2} \Phi^{\prime(2)}$

$=-\left[\left\{\left(h_{\rho}^{(1)} \Phi_{\rho}^{\prime(3)}+h_{\rho}^{(2)} \Phi_{\rho}^{\prime(2)}+h_{\rho}^{(3)} \Phi_{\rho}^{\prime(1)}+h_{z}^{(1)} \Phi_{z}^{\prime(3)}+h_{z}^{(2)} \Phi_{z}^{\prime(2)}+h_{z}^{(3)} \Phi_{z}^{\prime(1)}\right)\right.\right.$

$\left.\left.-\rho^{-1}\left(w_{\rho}^{(1)} \Phi_{z}^{(3)}+w_{\rho}^{(2)} \Phi_{z}^{(2)}+w_{\rho}^{(3)} \Phi_{z}^{(1)}-w_{z}^{(1)} \Phi_{\rho}^{(3)}-w_{z}^{(2)} \Phi_{\rho}^{(2)}-w_{z}^{(3)} \Phi_{\rho}^{(1)}\right)\right\}\right]$,

$\nabla^{2} \Phi^{(4)}+h^{(1)} \nabla^{2} \Phi^{(3)}+h^{(2)} \nabla^{2} \Phi^{(2)}$

$=-\left[\left\{\left(h_{\rho}^{(1)} \Phi_{\rho}^{(3)}+h_{\rho}^{(2)} \Phi_{\rho}^{(2)}+h_{\rho}^{(3)} \Phi_{\rho}^{(1)}+h_{z}^{(1)} \Phi_{z}^{(3)}+h_{z}^{(2)} \Phi_{z}^{(2)}+h_{z}^{(3)} \Phi_{z}^{(1)}\right)\right.\right.$

$\left.\left.-\rho^{-1}\left(w_{z}^{(1)} \Phi_{\rho}^{\prime(3)}+w_{z}^{(2)} \Phi_{\rho}^{\prime(2)}+w_{z}^{(3)} \Phi_{\rho}^{\prime(1)}-w_{\rho}^{(1)} \Phi_{z}^{\prime(3)}-w_{\rho}^{(2)} \Phi_{z}^{\prime(2)}-w_{\rho}^{(3)} \Phi_{z}^{\prime(1)}\right)\right\}\right]$.

Substituting the various expressions (and their derivatives, etc.) from the equations cited with some manipulations and taking terms up to $r^{-6}$, we have the following equations for (58a, b, c, d) :

$$
\begin{aligned}
& \nabla^{2} h^{(4)}=-2\left(B_{1}^{2}+B_{2}^{2}\right) r^{-4}+4\left(A_{1}+K_{1} B_{1}+K_{2} B_{2}\right)\left(B_{1}^{2}+B_{2}^{2}\right) r^{-5} \\
& -8\left(B_{1} B_{1}^{\prime}+B_{2} B_{2}^{\prime}\right) z r^{-6}+\frac{1}{2}\left[\left(A_{1}+K_{1} B_{1}+K_{2} B_{2}\right)^{4}\right. \\
& \left.+2\left(A_{1}+K_{1} B_{1}+K_{2} B_{2}\right)^{2}\left(B_{1}^{2}+B_{2}^{2}\right)+16\left(B_{1}^{2}+B_{2}^{2}\right)\right] r^{-6} \\
& +\left(A_{1}^{\prime}+K_{1} B_{1}^{\prime}+K_{2} B_{2}^{\prime}\right)\left(3 B_{1}^{2}+5 B_{2}^{2}\right) z r^{-7}-6\left(B_{1}^{\prime 2}+B_{2}^{\prime 2}\right) z^{2} r^{-8}+\ldots \ldots \ldots \\
& \Delta w^{(4)}=\left\{\frac{12}{5}\left(A_{1}+K_{1} B_{1}+K_{2} B_{2}\right)^{2}\left(B_{1} B_{2}^{\prime}-B_{1}^{\prime} B_{2}\right)\right. \\
& -\frac{24}{5} A_{2}\left(A_{1}+K_{1} B_{1}+K_{2} B_{2}\right)\left(B_{1}^{2}+B_{2}^{2}\right)+\frac{4}{5} A_{2}\left(A_{1}+K_{1} B_{1}+K_{2} B_{2}\right)^{3} \\
& \left.+8\left(B_{1}^{2}+B_{2}^{2}\right)\left(B_{1} B_{2}^{\prime}-B_{1}^{\prime} B_{2}\right)\right\} \rho^{2} r^{-8}-\left\{6\left(A_{1}+K_{1} B_{1}+K_{2} B_{2}\right)^{2}\left(B_{1} B_{2}^{\prime}-B_{1}^{\prime} B_{2}\right)\right. \\
& \left.-2 A_{2}\left(A_{1}+K_{1} B_{1}+K_{2} B_{2}\right)\left(B_{1}^{2}+B_{2}^{2}\right)+2 A_{2}\left(A_{1}+K_{1} B_{1}+K_{2} B_{2}\right)^{3}\right\} \rho^{4} r^{-10} \\
& -\left\{2 A_{2}\left(A_{1}+K_{1} B_{1}+K_{2} B_{2}\right)^{3}+2\left(A_{1}+K_{1} B_{1}+K_{2} B_{2}\right)^{2}\left(B_{1} B_{2}^{\prime}-B_{1}^{\prime} B_{2}\right)\right. \\
& \left.-2 A_{2}\left(A_{1}+K_{1} B_{1}+K_{2} B_{2}\right)\left(B_{1}^{2}+B_{2}^{2}\right)+8\left(B_{1}^{2}+B_{2}^{2}\right)\left(B_{1} B_{2}^{\prime}-B_{1}^{\prime} B_{2}\right)\right\} \rho^{2} z^{2} r^{-10}
\end{aligned}
$$

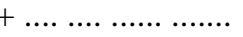

$$
\begin{aligned}
& \nabla^{2} \Phi^{\prime(4)}=-\left[\frac{1}{2} B_{2}\left(A_{1}+K_{1} B_{1}+K_{2} B_{2}\right)^{3}\right. \\
& \left.+\left(A_{1}+K_{1} B_{1}+K_{2} B_{2}\right)\left(11 B_{1}^{2} B_{2}+5 B_{2}^{3}\right)\right] r^{-6}+ \\
& \nabla^{2} \Phi^{(4)}=-\left[\frac{1}{2} B_{1}\left(A_{1}+K_{1} B_{1}+K_{2} B_{2}\right)^{3}\right. \\
& \left.+8 B_{1}\left(B_{1}^{2}+B_{2}^{2}\right)\left(A_{1}+K_{1} B_{1}+K_{2} B_{2}\right)\right] r^{-6}+
\end{aligned}
$$

Now we shall find the solutions of the foregoing equations :

From (59a), we have

$\nabla^{2} h^{(4)}=l_{1} r^{-4}+l_{2} r^{-5}+l_{3} z r^{-6}+l_{4} r^{-6}+l_{5} z r^{-7}+l_{6} z^{2} r^{-8}$

where $l_{1}, l_{2}, l_{3}, l_{4}, l_{5}$ and $l_{6}$ are constants and co-efficients of $r^{-4}, r^{-5}, z r^{-6}, r^{-6}, z r^{-7}$ and $z^{2} r^{-8}$ respectively (It will be clear from the context that in some cases we omit the dots, i.e., terminate the expression). 
Let the solution of (60) be

$h^{(4)}=m_{1} r^{-2}+m_{2} r^{-3}+m_{3} z r^{-4}+m_{4} r^{-6}+m_{5} z r^{-5}+m_{6} z^{2} r^{-6}$,

where $m_{1}, m_{2}, m_{3}, m_{4}, m_{5}$ and $m_{6}$ are constants, to be determined.

Applying $\nabla^{2}$ in (61), we get

$\nabla^{2} h^{(4)}=m_{1} \nabla^{2} r^{-2}+m_{2} \nabla^{2} r^{-3}+m_{3} \nabla^{2} z r^{-4}+m_{4} \nabla^{2} r^{-6}+m_{5} \nabla^{2} z r^{-5}+m_{6} \nabla^{2} z^{2} r^{-6}$.

Now using the following equation

$$
\begin{aligned}
\left(\rho^{n} z^{m} r^{-p}\right)_{\rho} & =z^{m}\left(n \rho^{n-1} r^{-p}-p \rho^{n+1} r^{-p-2}\right),\left(\rho^{n} z^{m} r^{-p}\right)_{z}=\rho^{n}\left(m z^{m-1} r^{-p}-p z^{m+1} r^{-p-2}\right), \\
\left(\rho^{n} z^{m} r^{-p}\right)_{\rho \rho} & =z^{m}\left[n(n-1) \rho^{n-2} r^{-p}-p(2 n+1) \rho^{n} r^{-p-2}+p(p+2) \rho^{n+2} r^{-p-4}\right], \\
\left(\rho^{n} z^{m} r^{-p}\right)_{z z} & =\rho^{n}\left[m(m-1) z^{m-2} r^{-p}-p(2 m+1) z^{m} r^{-p-2}+p(p+2) z^{m+2} r^{-p-4}\right], \\
\nabla^{2}\left(\rho^{n} z^{m} r^{-p}\right) & =\left(\rho^{n} z^{m} r^{-p}\right)_{\rho \rho}+\left(\rho^{n} z^{m} r^{-p}\right)_{z z}+\rho^{-1}\left(\rho^{n} z^{m} r^{-p}\right)_{\rho} \\
& =\left[n^{2} \rho^{n-2} z^{m} r^{-p}+m(m-1) \rho^{n} z^{m-2} r^{-p}+p(p-2 n-2 m-1) \rho^{n} z^{m} r^{-p-2}\right], \\
\Delta\left(\rho^{n} z^{m} r^{-p}\right) & =\left(\rho^{n} z^{m} r^{-p}\right)_{\rho \rho}+\left(\rho^{n} z^{m} r^{-p}\right)_{z z}-\rho^{-1}\left(\rho^{n} z^{m} r^{-p}\right) \rho \\
& =\left[n(n-2) \rho^{n-2} z^{m} r^{-p}+m(m-1) \rho^{n} z^{m-2} r^{-p}+p(p-2 n-2 m+1) \rho^{n} z^{m} r^{-p-2}\right]
\end{aligned}
$$

(eq. (38) Salam (2016)) [8] into equation (62) and by some simplifications, we get

$$
\nabla^{2} h^{(4)}=2 m_{1} r^{-4}+6 m_{2} r^{-5}+4 m_{3} z r^{-6}+\left(12 m_{4}+2 m_{6}\right) r^{-6}+10 m_{5} z r^{-7}+6 m_{6} z^{2} r^{-8} \text {. }
$$

Since (63) and (60) are identical, therefore comparing the aforesaid equations, we have

$$
m_{1}=\frac{1}{2} l_{1}, m_{2}=\frac{1}{6} l_{2}, m_{3}=\frac{1}{4} l_{3}, m_{4}=\frac{1}{12}\left(l_{4}-\frac{1}{3} l_{6}\right), m_{5}=\frac{1}{10} l_{5} \text { and } m_{6}=\frac{1}{6} l_{6} \text {. }
$$

Using the above values (64) into (63) and by simple simplification, we get

$$
\nabla^{2} h^{(4)}=l_{1} r^{-4}+l_{2} r^{-5}+l_{3} z r^{-6}+l_{4} r^{-6}+l_{5} z r^{-7}+l_{6} z^{2} r^{-8} \text {. }
$$

Since the final form (65) is the same as (60); therefore (61) is the solution of (60).

From (59b), we have

$$
\Delta w^{(4)}=l_{1}^{\prime} \rho^{2} r^{-8}+l_{2}^{\prime} \rho^{4} r^{-10}+l_{3}^{\prime} \rho^{2} z^{2} r^{-10},
$$

where $l_{1}^{\prime}, l_{2}^{\prime}, l_{3}^{\prime}$ are constants and co-efficients of $\rho^{2} r^{-8}, \rho^{4} r^{-10}$ and $\rho^{2} z^{2} r^{-10}$ respectively.

Let the solution of (66) be

$$
w^{(4)}=m_{1}^{\prime} \rho^{2} r^{-6}+m_{2}^{\prime} \rho^{4} r^{-8}+m_{3}^{\prime} \rho^{2} z^{2} r^{-8},
$$

where $m_{1}^{\prime}, m_{2}^{\prime}, m_{3}^{\prime}$ are constants, to be determined.

Applying $\Delta$ on both sides of (67), we get

$\Delta w^{(4)}=m_{1}^{\prime} \Delta \rho^{2} r^{-6}+m_{2}^{\prime} \Delta \rho^{4} r^{-8}+m_{3}^{\prime} \Delta \rho^{2} z^{2} r^{-8}$.

Making use of (63a) into the equation (68) and by some manipulation, we have

$$
\Delta w^{(4)}=\left(18 m_{1}^{\prime}+8 m_{2}^{\prime}+2 m_{3}^{\prime}\right) \rho^{2} r^{-8}+8 m_{2}^{\prime} \rho^{4} r^{-10}+8 m_{3}^{\prime} \rho^{2} z^{2} r^{-10} \text {. }
$$

Since (69) and (66) are identical, so equating the co-efficients of like terms, we have

$$
m_{1}^{\prime}=\frac{1}{18}\left(l_{1}^{\prime}-l_{2}^{\prime}-\frac{1}{4} l_{3}^{\prime}\right), m_{2}^{\prime}=\frac{1}{8} l_{2}^{\prime}, m_{3}^{\prime}=\frac{1}{8} l_{3}^{\prime}
$$

Substituting the above values of (70) into (69), we obtain

$$
\Delta w^{(4)}=l_{1}^{\prime} \rho^{2} r^{-8}+l_{2}^{\prime} \rho^{4} r^{-10}+l_{3}^{\prime} \rho^{2} z^{2} r^{-10} \text {. }
$$

Here (71) is the same as (66). Hence (67) is the solution of (66). 
From $(59 \mathrm{c})$, we have

$\nabla^{2} \Phi^{\prime(4)}=\alpha_{1}^{\prime} r^{-6}+$

where

$\alpha_{1}^{\prime}=-\left[\frac{1}{2} B_{2}\left(A_{1}+K_{1} B_{1}+K_{2} B_{2}\right)^{3}+\left(A_{1}+K_{1} B_{1}+K_{2} B_{2}\right)\left(11 B_{1}^{2} B_{2}+5 B_{2}^{3}\right)\right]$.

Let the solution of (72) be

$\Phi^{\prime(4)}=\beta_{1}^{\prime} r^{-4}$,

where $\beta_{1}^{\prime}$ is a constant, to be determined.

From (73), we have

$\nabla^{2} \Phi^{\prime(4)}=\beta_{1}^{\prime} \nabla^{2} r^{-4}$

Using (63a) in the above equation (74), we obtain

$\nabla^{2}\left(r^{-4}\right)=12 r^{-6}$

Putting (75) into (74) we have

$\nabla^{2} \Phi^{\prime(4)}=12 \beta_{1}^{\prime} r^{-6}$.

Since (76) and (72) are identical, therefore by comparing the two, we get

$\beta_{1}^{\prime}=\frac{1}{12} \alpha_{1}^{\prime}$.

Putting the value of $\beta_{1}^{\prime}$ into (76), we have

$\nabla^{2} \Phi^{\prime(4)}=\alpha_{1}^{\prime} r^{-6}$

which is the same as (72). Hence (73) is the solution of (72).

Again from (59d), we obtain

$\nabla^{2} \Phi^{(4)}=\alpha_{2}^{\prime} r^{-6}+$

where, $\alpha_{2}^{\prime}=-\left[\frac{1}{2} B_{1}\left(A_{1}+K_{1} B_{1}+K_{2} B_{2}\right)^{3}+8 B_{1}\left(B_{1}^{2}+B_{2}^{2}\right)\left(A_{1}+K_{1} B_{1}+K_{2} B_{2}\right)\right]$.

Let the solution of (79) be

$\Phi^{(4)}=\beta_{2}^{\prime} r^{-4}$,

where, $\beta_{2}^{\prime}$ is a constant.

From (80), we have

$\nabla^{2} \Phi^{(4)}=\beta_{2}^{\prime} \nabla^{2} r^{-4}$

Making use of (63a), we find

$\nabla^{2} r^{-4}=12 r^{-6}$.

Putting (82) into (81), we get

$\nabla^{2} \Phi^{(4)}=12 \beta_{2}^{\prime} r^{-6}$.

Comparing (83) with (79), we find

$\beta_{2}^{\prime}=\frac{1}{12} \alpha_{2}^{\prime}$.

Substituting the value of $\beta_{2}^{\prime}$ into (83), we have

$\nabla^{2} \Phi^{(4)}=\alpha_{2}^{\prime} r^{-6}$

which is the same as (79). Therefore (80) is the solution of (79) [7]. 


\section{Acknowledgments}

I am indebted to my most respected supervisor Late Emeritus Professor Dr. J. N. Islam Ph.D., Sc. D. (Cantab) whose whole-hearted cooperation and valuable advice necessary for the completion of this work. I am also indebted to Professor Dr. M. A. M. Chowdhury former Director, JNIRCMPS (C.U.) for many healpfull discussions.

\section{References}

[1]. Salam, M.A., Ganit: J. Bangladesh Math. Soc. 20, 1-14 (Paper II) (2000)

[2]. Salam, M.A., Ganit: J. Bangladesh Math. Soc. 17, 37-47 (Paper I) (1997).

[3]. Salam, M.A., Studies of An Approximation Scheme for Rotating Solutions of Einstein's Equations, M. Phil. Thesis. University of Chittagong. (1988).

[4]. Islam, J.N., Math. Proc. Camb. Phil. Soc. 79, 161 (1976a).

[5]. Islam, J.N., Gen. Rel. Grav. 7, 669 (1976b)

[6]. Islam, J.N., Rotating Fields in General Relativity (Cambridge University Press). (1985).

[7]. Salam, M.A., Studies in the solution of Rotating Fields in General Relativity, Ph.D. Thesis, University of Chittagong (2010).

[8]. Salam, M. A., IOSR Journal of Mathematics (IOSR-JM) Volume 12, Issue 2 Ver I (Mar.-Apr. 2016), PP 18-32 (Paper III)

[9]. Papapetrou, A. Proc. Roy. Irish Acad., A51, 191. (1947)

[10]. Majumdar, S.D., Phys. Rev., 72, 390. (1947)

[11]. Weyl, H., Ann. Physik, 54, 117. (1917)

[12]. Perjes, Z., Phys. Rev., Lett., 27, 1668. (1971)

[13]. Israel, W., and Wilson, G.A., J. Math. Phys., 13, 865. (1972)

[14]. Bonnor, W.B., Commun. Math. Phys., 34, 77. (1973)

[15]. Ernst, F.J., Phys. Rev., 168, 1415. (1968b)

[16]. Misner, C.W., Thorne, K.S., and Wheeler, J.A., Gravitation, W.H. Freeman, San Francisco (1973). 